PROCEEDINGS OF THE

AMERICAN MATHEMATICAL SOCIETY

Volume 142, Number 11, November 2014, Pages 3757-3760

S 0002-9939(2014)12203-9

Article electronically published on July 24, 2014

\title{
POWER SETS AND SOLUBLE SUBGROUPS
}

\author{
MARTIN W. LIEBECK AND ANER SHALEV \\ (Communicated by Pham Huu Tiep)
}

\begin{abstract}
We prove that for certain positive integers $k$, such as 12 , a normal subgroup of a finite group which consists of $k^{\text {th }}$ powers is necessarily soluble. This gives rise to new solubility criteria, and solves an open problem from a 2013 paper by the authors.
\end{abstract}

\section{INTRODUCTION}

For a group $G$ and a positive integer $k$, denote by $G^{[k]}$ the set $\left\{x^{k}: x \in G\right\}$ of $k^{\text {th }}$ powers in $G$. Define a positive integer $k$ to be nice if $k$ is a multiple of one of the following numbers:

$2^{a} p$, where $a>1$ and $p$ is a prime divisor of $2^{2^{a+1}}-1$,

$2 \cdot 3^{a} p$, where $a \geq 1$ and $p$ is an odd prime divisor of $3^{3^{a}} \pm 1$,

$3^{a} \cdot 5 p$, where $a \geq 1$ and $p$ is an odd prime divisor of $3^{3^{a}} \pm 1$.

Note that the smallest few nice numbers are multiples of 12, 20, 42, 68, 78, 105.

Theorem 1. Let $G$ be a finite group and suppose $N$ is a normal subgroup of $G$ contained in $G^{[k]}$ for some nice integer $k$. Then $N$ is soluble.

Corollary 2. Let $k$ be a nice integer and suppose $G$ is a finite group such that $G^{[k]}$ contains a subgroup $H$ of index $c$ in $G$. Then $G$ has a soluble normal subgroup of index dividing $c$ !

Indeed, the core of $H$ is the required normal subgroup, by Theorem 1 Corollary 2 is in the same spirit as [1, where certain infinite groups $G$ with the property that $G^{[k]}$ contains a subgroup of finite index in $G$ are studied.

Corollary 3. Suppose $k$ is a nice integer and $G$ is a finite group such that $\operatorname{Aut}(G)^{[k]}$ contains $\operatorname{Inn}(G)$. Then $G$ is soluble.

This follows immediately from Theorem 1 since $\operatorname{Inn}(G)$ is normal in $\operatorname{Aut}(G)$ the theorem implies that $\operatorname{Inn}(G)$ is soluble, hence so is $G$.

In 2, some solubility criteria for finite groups are established, and Theorem 1 implies some of them. For example, if $G^{[12]}$ is a subgroup of $G$, then Theorem 1 implies that $G^{[12]}$ is soluble, and since $G / G^{[12]}$ is also soluble (by Burnside's $p^{a} q^{b}$ theorem), $G$ is soluble as well, which is [2, Theorem 1].

Theorem 1 is proved via the following two results.

Received by the editors December 20, 2012.

2010 Mathematics Subject Classification. Primary 20D10, 20E07, 20 D06.

The authors are grateful for the support of an EPSRC grant.

The second author acknowledges the support of Advanced ERC Grant 247034, an ISF grant 754/08, and the Miriam and Julius Vinik Chair in Mathematics, which he holds.

(C)2014 American Mathematical Society Reverts to public domain 28 years from publication 
Proposition 4. Let $k$ be a positive integer, and suppose that there is no non-abelian finite simple group $T$ such that $(\operatorname{Aut}(T))^{[k]}$ contains $T$. Then any normal subgroup of a finite group which consists of $k^{\text {th }}$ powers is soluble.

Proposition 5. A number $k$ is nice if and only if there is no non-abelian finite simple group $T$ such that $(\operatorname{Aut}(T))^{[k]}$ contains $T$.

A main problem posed in [2, Section 4] is the characterization of positive integers $k$ with the property that finite groups $G$ for which $G^{[k]}$ is a subgroup are all soluble. Our last result solves this problem.

Theorem 6. For a positive integer $k$, the following two conditions are equivalent:

(i) Every finite group $G$ such that $G^{[k]}$ is a subgroup is soluble.

(ii) Either

(a) $k$ is an odd number of the form $3^{a} 5^{b} m$, where $a, b \geq 1$ and $\left(m, 3^{2 \cdot 3^{a}}-1\right) \neq 1$, or

(b) $k$ is an even number which is not one of the following:

$(\alpha)$ a multiple of $\exp (T)$, the exponent of some finite non-abelian simple group $T$,

(ß) $2 \cdot 3^{a} \cdot m$, where $a \geq 0$ and $\left(m, 3\left(3^{2 \cdot 3^{a}}-1\right)\right)=1$,

$(\gamma) 2^{a} \cdot m$, where $a \geq 2$ and $\left(m, 2\left(2^{2^{a+1}}-1\right)\right)=1$.

\section{Proof of Theorem 1}

Proof of Proposition 4. Let $k$ be as in the statement of the proposition and suppose $G$ is a minimal counterexample. So $G$ has an insoluble normal subgroup $N$ consisting of $k^{t h}$ powers. Let $M$ be a minimal normal subgroup of $G$. Then $N M / M$ is soluble. If $M_{1}$ is another minimal normal subgroup of $G$, then $G$ embeds in $G / M \times G / M_{1}$ and so $N$ is soluble, a contradiction. Hence $M$ is the unique minimal normal subgroup of $G$ and $M \leq N$. Moreover $M$ is non-abelian (otherwise $N$ would be soluble), so $C_{G}(M)=1, M=T^{r}$ for some non-abelian simple group $T$, and $G$ embeds in $\operatorname{Aut}(M)=\operatorname{Aut}(T) \imath S_{r}$.

By the choice of $k,(\operatorname{Aut}(T))^{[k]}$ does not contain $T$, and so there exists $t \in$ $T \backslash \operatorname{Aut}(T)^{[k]}$. We claim that the element $n=(t, 1, \ldots, 1) \in T^{r}=M$ is not a $k^{t h}$ power in $G$. To see this, suppose $n=x^{k}$, where $x=\left(x_{1}, \ldots, x_{r}\right) \sigma$ with each $x_{i} \in \operatorname{Aut}(T)$ and $\sigma \in S_{r}$. Then $\sigma^{k}=1$. If $\sigma(1)=1$, then $t=x_{1}^{k}$, contradicting the fact that $t$ is not a $k^{t h}$ power in $\operatorname{Aut}(T)$. So $\sigma$ has a cycle $\left(1 i_{2} \cdots i_{s}\right)$ with $s \geq 1$. Calculating the coordinates of $x^{k}$ in positions 1 and $i_{s}$, we get $t=x_{1} x_{i_{2}} \cdots x_{i_{s}}$ and $1=x_{i_{s}} x_{1} \cdots x_{i_{s-1}}$, a contradiction.

It follows from the claim that $G^{[k]}$ does not contain $M$, which is a contradiction since $M \leq N \subseteq G^{[k]}$. This completes the proof.

Proof of Proposition 5. The main tool for this proof is the following result from [2].

Theorem 7 ([2, Propositions 5, 6, and Theorem 7]). Let $T$ be a finite simple group, and let $m>1$ be a positive integer dividing $|T|$. Suppose $\operatorname{Aut}(T)^{[m]}$ contains $T$. Then $m=p^{r}$ or $2 p^{r}$ for some prime $p$. Further, if $m=2$, then $T=L_{2}(q)$ ( $q$ odd), $L_{2}\left(q^{2}\right)$ (q even) or $L_{3}(4)$; and if $m=p^{r}>2$ or $m=2 p^{r}$ ( $p$ odd), then $T=L_{2}\left(p^{m l}\right)$ or $L_{2}\left(p^{m l / 2}\right)$, respectively. Conversely, $\operatorname{Aut}(T)^{[m]}$ contains $T$ for all such $T$ and $m$.

We embark on the proof of Proposition 5 . 
Suppose $k>1$ is an integer which is nice. Assume for a contradiction that there exists a non-abelian simple group $T$ such that $\operatorname{Aut}(T)^{[k]}$ contains $T$. Then $\operatorname{Aut}(T)^{[m]}$ contains $T$ for any divisor $m$ of $k$, so we may assume that $k$ is one of the numbers $2^{a} p, 2 \cdot 3^{a} p, 3^{a} \cdot 5 p$ as in the definition of nice numbers.

Consider $k=2^{a} p$ with $a>1$ and $p$ a prime divisor of $2^{2^{a+1}}-1$. Certainly 4 divides $|T|$, so Theorem 7 implies that $T=L_{2}\left(2^{4 l}\right)$ for some $l$. This is then divisible by $2^{3}$, so if $a \geq 3$, Theorem 7 gives $T=L_{2}\left(2^{2^{3} l^{\prime}}\right)$ for some $l^{\prime}$. Repeating this argument, we see that $T=L_{2}\left(2^{2^{a} l^{\prime \prime}}\right)$ for some $l^{\prime \prime}$. But then $p$ divides $|T|$ and $\operatorname{Aut}(T)^{[p]}$ contains $T$, which is a contradiction by Theorem 7 .

Now consider $k=2 \cdot 3^{a} p$, where $a \geq 1$ and $p$ is an odd prime divisor of $3^{3^{a}} \pm 1$. Since $\operatorname{Aut}(T)^{[2]}$ contains $T$, Theorem 7 gives $T=L_{2}(q)$ or $L_{3}(4)$. In particular, 3 divides $|T|$, so again by Theorem $7, T=L_{2}\left(3^{3 l}\right)$, and arguing as before, $T=$ $L_{2}\left(3^{3^{a} l^{\prime}}\right)$. Then $p$ divides $|T|$, giving a contradiction by Theorem 7

Finally, consider $k=3^{a} \cdot 5 p$, where $a \geq 1$ and $p$ is an odd prime divisor of $3^{3^{a}} \pm 1$. If 3 does not divide $|T|$, then $T$ is a Suzuki group, but then 5 divides $|T|$ and $\operatorname{Aut}(T)^{[5]}$ contains $T$, contrary to Theorem 7. Hence 3 divides $|T|$ and so $T=L_{2}\left(3^{3 l}\right)$. Now argue as in the previous paragraph. This proves one implication in Proposition 5 and already establishes Theorem 1 .

For the converse implication of Proposition 5, assume that $k>1$ is not nice. We need to find a non-abelian simple group $T$ such that $\operatorname{Aut}(T)^{[k]}$ contains $T$.

First consider the case where $k$ is odd. If $(k, 3)=1$, one can see using Dirichlet's theorem on primes in arithmetic progession that there is a prime $p>3$ such that $T=L_{2}(p)$ has order coprime to $k$, and hence $T^{[k]}=T$. Also, if $(k, 5)=1$, then there is a large prime $p$ such that the Suzuki group $T={ }^{2} B_{2}\left(2^{p}\right)$ has order coprime to $k$, giving the same conclusion. Hence we may assume that 15 divides $k$. Let $k=3^{a} 5^{b} m$ with $m$ coprime to 15 . As $k$ is not nice, we have $\left(m, 3^{3^{a}} \pm 1\right)=1$. Then the group $T=L_{2}\left(3^{3^{a}}\right)$ has order coprime to $5^{b} m$ and hence satisfies $T \subseteq \operatorname{Aut}(T)^{[k]}$ by Theorem 7 .

Now assume $k$ is even and divisible by 4 , and write $k=2^{a} m$ with $m$ odd. As $k$ is not nice, $\left(m, 2^{2^{a+1}}-1\right)=1$. Then by Theorem 7 we have $T \subseteq \operatorname{Aut}(T)^{[k]}$ for $T=L_{2}\left(2^{2^{a}}\right)$.

Finally, assume $k=2 l$ with $l$ odd. If $(k, 3)=1$, then we can find a prime $p>3$ such that $l$ is coprime to the order of $T=L_{2}(p)$, and then $T \subseteq \operatorname{Aut}(T)^{[k]}$ by Theorem 7 . So assume 3 divides $k$ and write $k=2 \cdot 3^{a} m$ with $m$ coprime to 6 . As $k$ is not nice, $\left(m, 3^{3^{a}} \pm 1\right)=1$. But then $T \subseteq \operatorname{Aut}(T)^{[k]}$ for $T=L_{2}\left(3^{3^{a}}\right)$. This completes the proof of Proposition 5 .

\section{Proof of Theorem 6}

As in [2, define a positive integer $k$ to be good if it satisfies condition (i) of Theorem 6, and bad otherwise.

First let $k$ be an odd integer. If $k$ is coprime to 3 or 5 , then as above, there is a simple group $T=L_{2}(p)$ or ${ }^{2} B_{2}(q)$ of order coprime to $k$, and then $T^{[k]}=T$, showing that $k$ is bad. So assume $k=3^{a} 5^{b} m$ with $a, b \geq 1$ and $m$ coprime to 15. If $\left(m, 3^{3^{a}} \pm 1\right)=1$, then $T=L_{2}\left(3^{3^{a}}\right)$ has order coprime to $5^{b} m$, and also, by [2, Proposition 6], $G=T\langle\sigma\rangle$ satisfies $G^{[k]}=T$ for a field automorphism $\sigma$; hence $k$ is bad. On the other hand, if $\left(m, 3^{3^{a}} \pm 1\right) \neq 1$, then we claim that $k$ is good. 
Suppose $G$ is a finite group such that $G^{[k]}$ is a subgroup, and suppose $G$ has a nonabelian composition factor $T$. By [2, Theorem 4], we have $T \subseteq \operatorname{Aut}(T)^{[k]}$. Hence we can use Theorem 7 as before to see that $T$ must be $L_{2}\left(3^{3^{a} l}\right)$ for some $l$. But if $p$ is a prime divisor of $\left(m, 3^{3^{a}} \pm 1\right)$, then $p$ divides $|T|$, so $T \subseteq \operatorname{Aut}(T)^{[p]}$, which is a contradiction by Theorem 7 . Hence $G$ is soluble and so $k$ is good, proving the claim.

We have now shown that the odd good numbers are precisely those in (a) of Theorem 6

Now let $k$ be even. Of course if $k$ is a multiple of the exponent $\exp (T)$ of a simple group $T$, then $k$ is bad.

Assume 4 divides $k$, and write $k=2^{a} m$ with $a \geq 2$ and $m$ odd. If $\left(m, 2^{2^{a+1}}-1\right)$ $=1$, then for $T=L_{2}\left(2^{2^{a}}\right)$, the group $G=T\langle\sigma\rangle$, where $\sigma$ is a field automorphism of order $2^{a}$, satisfies $G^{[k]}=T$ (see [2, Proposition 6]), so $k$ is bad. On the other hand, if $\left(m, 2^{2^{a+1}}-1\right) \neq 1$, then the argument given above for odd numbers shows that $k$ is good.

Finally, assume that $k=2 l$ with $l$ odd. If $l$ is coprime to 3 , then there is a prime $p>3$ such that $L_{2}(p)$ has order coprime to $l$, and $G=P G L_{2}(p)$ satisfies $G^{[k]}=L_{2}(p)$ (see 2, Proposition 5]), so $k$ is bad. Now assume 3 divides $l$, and write $k=2 \cdot 3^{a} \cdot m$ with $m$ coprime to 6 . If $\left(m, 3^{2 \cdot 3^{a}}-1\right)=1$, then the group $G=L_{2}\left(3^{3^{a}}\right)\langle\sigma\rangle$ satisfies $G^{[k]}=T$; otherwise, the usual argument shows that $k$ is good. This completes the proof of Theorem [6]

\section{REFERENCES}

[1] E. Hrushovski, P. H. Kropholler, A. Lubotzky, and A. Shalev, Powers in finitely generated groups, Trans. Amer. Math. Soc. 348 (1996), no. 1, 291-304, DOI 10.1090/S0002-9947-9601456-0. MR1316851 (96f:20061)

[2] M. W. Liebeck and A. Shalev, Powers in finite groups and a criterion for solubility, Proc. Amer. Math. Soc. 141 (2013), no. 12, 4179-4189. MR3105861

Department of Mathematics, Imperial College, London SW7 2AZ, United Kingdom

Institute of Mathematics, Hebrew University, Jerusalem 91904, Israel 